\title{
Delayed Presentation of Catheter-related Subclavian Artery Pseudoaneurysm
}

\author{
Kim $M,{ }^{1}$ Kang ES, ${ }^{1}$ Kim HW, ${ }^{1}$ Kim $Y,{ }^{2}$ Kang $M H^{,},{ }^{2}$ Chang $Y J,{ }^{\prime}$ Choe KH, Lee KM, An JY' \\ 'Department of Internal Medicine, ${ }^{2}$ The Radiology, College of Medicine, Chungbuk National University, Cheong-ju, Republic of \\ Korea.
}

\section{ABSTRACT}

Central venous catheterization is a common diagnostic and therapeutic procedure in modern clinical practice. Pseudoaneurysms of the subclavian artery are rare and usually occur immediately after the causative event, whether the cause was trauma or a medical procedure. Here, we report a case of a 71-year-old woman with delayed presentation of catheter-related subclavian pseudoaneurysm. The patient's symptoms began two weeks after the initial catheterization, probably because of slow leakage of blood from the injured subclavian artery caused by incomplete compression of the puncture site and uremic coagulopathy. She was successfully treated with ultrasound-guided thrombin and angiography-guided histoacryl injection without stent insertion or surgery.

Keywords: butyl 2-cyanacrylate; pseudoaneurysm; subclavian; thrombin.

\section{INTRODUCTION}

Central venous catheters are commonly used for diagnostic and therapeutic purposes. However, they may sometimes be the cause of a number of catheterrelated complications ranging from mild to severe. ${ }^{1}$ Rare, potentially serious complications include development of a pseudoaneurysm of the subclavian artery and its subsequent progression and eventual rupture. Most of the complications occur and present immediately after the causative event, but in rare cases presentation could be delayed. Here we report the rare case of a 71-yearold woman who showed a delayed presentation of catheter-related subclavian pseudoaneurysm and was successfully treated with ultrasound-guided thrombin and angiography-guided histoacryl injection.

\section{CASE REPORT}

A 71-year-old woman was transferred to the emergency department for specialized intensive care including renal replacement therapy. Her medical history included diabetes and a previous cerebrovascular incident. She had been admitted at another hospital one month earlier because of aspiration pneumonia. Although broadspectrum antibiotics were administered, her condition had gradually worsened. Eventually, she was intubated for respiratory failure. Her doctor had attempted to place a central line via the left subclavian vein. Vessel access was performed using the standard needle and guidewire technique without ultrasonographic guidance, but catheter placement via the left subclavian vein failed even after several trials. Even with aggressive treatment, her symptoms gradually worsened, and she developed acute kidney injury. She was transferred to our hospital for continuous renal replacement therapy. In the emergency department, laboratory diagnostics revealed the following: leukocyte count, $22,160 / \mathrm{mm}^{3}$; C-reactive protein level, $3.95 \mathrm{mg} / \mathrm{dL}$; and pro-calcitonin quantitative level, $2.15 \mu \mathrm{g} / \mathrm{L}$. The other blood chemistry values were within reference ranges except increased blood urea nitrogen $(70 \mathrm{mg} / \mathrm{dL})$ and creatinine $(4.7 \mathrm{mg} /$ $\mathrm{dL}$ ). In order to protect her renal function, we changed her antibiotic to a less nephrotoxic one with a dose adjustment. Fortunately, her respiratory symptoms improved along with her renal function. However, on the fifth day after she was transferred to our hospital, we found swelling in her left upper anterior chest area. We performed a computed tomography (CT) examination

Correspondence: Jin-Young An, Department of Internal Medicine, Chungbuk National University Hospital ,776, 1 sunhwan-ro, Seowon-gu, Cheongiu-si, Chungcheongbuk-do 361-711, Korea. Phone: 82-043-2696308, Fax :82-043-269-6804. 
of the chest. CT scanning revealed a $2.6-\mathrm{cm}$ enhancing nodular lesion, suggesting a pseudoaneurysm at the left subclavicular area with surrounding hematoma (Figure 1a). We immediately performed an ultrasound-guided thrombin injection at the pseudoaneurysm of the left subclavian artery (Figure 2). The swelling gradually decreased over the next four days. On the eighth day after the procedure, swelling of the chest wall had begun to increase, and we performed ultrasonography. On ultrasonography, blood flow could not be visualized at the pseudoaneurysm because the vessel was blocked by a thrombus. Angiography was performed to find the cause of the swelling, and embolization was performed at the same branch of the subclavian arteryusingnButyl-2-Cyanoacrylate (Histoacryl, B. Braun Medical Inc., Bethlehem, PA, USA)(Figure 3a). Follow-up angiography, performed after four days, revealed no visible extravasation from the subclavian artery(Fig: 3b).

Both the patient's general condition and left chest swelling gradually improved. Follow-up CT scanning revealed non-visualization of active bleeding and pseudoaneurysm (Fig: 1B). One month later, she was discharged to home.

\section{DISCUSSION}

A central venous line may be greatly useful in monitoring the cardiovascular function of patients in critical condition and as a route to administer vasoactive drugs or solutions that would irritate peripheral veins, such as total parenteral nutrition. ${ }^{2}$ However, central line insertion may cause a number of complications such as skin infection, thrombophlebitis, pneumothorax, thrombosis, central-line-associated sepsis, hemorrhage, and arrhythmia. ${ }^{1}$ Pseudoaneurysm of the subclavian artery can also develop after inadvertent puncture of the artery. Subclavian pseudoaneurysms are rare, and their true incidence is unknown. The majority of cases are secondary to arterial catheterization, surgical procedures, and radiology interventions, but in a few cases described in the literature, injury to the subclavian artery was caused by blunt trauma. ${ }^{3}$ Patients with subclavian pseudoaneurysm may be asymptomatic or they may present with chest pain, Horner syndrome, paresthesia, hoarseness, upper limb ischemia, a pulsatile mass, or hemoptysis. ${ }^{4}$

Early recognition and optimal diagnostic tools are very important in those cases. The following methods may be used to aid in diagnosis. Sonography is highly sensitive and specific for accessible arterial injuries, and it is often useful for rapid bedside assessment in the intensive care unit. Spiral contrast-enhanced computed tomography usually provides a good diagnostic yield and was used to make the initial diagnosis in this case. Angiography not only enables accurate diagnosis but also provides endovascular treatment options without surgery. ${ }^{5}$ These are complementary diagnostic tools, and their usage depends on patients' conditions.

One interesting aspect of our case described here was the presentation delayed for around two weeks after catheterization. We suggest the following possible explanations of the delayed presentation. First, the patient was a quiet, overweight, geriatric woman with ample free space for hematoma reservoir. Second, she could not complain of chest discomfort because of intubation and deep sedation during ventilator care. Third, uremic coagulopathy played a role in the continuous leakage of small amounts of blood from the injured vessel. ${ }^{6}$

The classical treatment of pseudoaneurysm is open surgery for resection and end-to-end anastomosis, venous graft, suture, or bypass. ${ }^{7}$ However, open surgery involves considerable morbidity and mortality, particularly with high-risk patients and urgent surgeries. Therefore, less invasive procedures have been developed. Marin et al. published the first report of the use of covered stents to treat pseudoaneurysms. ${ }^{8}$ Since the 1990s, ultrasound-guided compression repair and ultrasound-guided thrombin injection have been used. Ultrasound-guided thrombin injection has a primary success rate of $97 \%$ and a low complication rate of $1.3 \%$, with an embolic rate of $0.5 \% .{ }^{9}$ Moreover, use of endovascular stent treatment or embolization of vascular injuries via angiography has become an acceptable and less invasive alternative to surgical repair in subclavian artery injury. ${ }^{10}$ We have also successfully performed ultrasound-guided thrombin injection and histoacryl embolization by angiography without stent.

In conclusion, overweight and geriatric patients with uremic coagulopathy could be predisposed to bleeding and delayed presentation of hematoma and pseudoaneurysm. Therefore, such patients require accurate and relatively long compression times compared with other patients. Also, ultrasound-guided access should also be considered in order to avoid unnecessary damage to blood vessels, if it is available for use. 


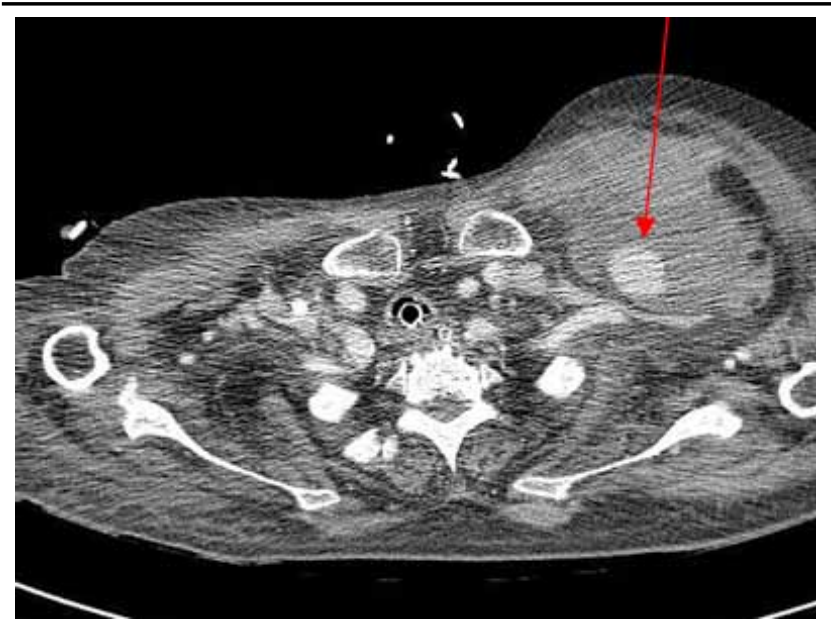

(A)

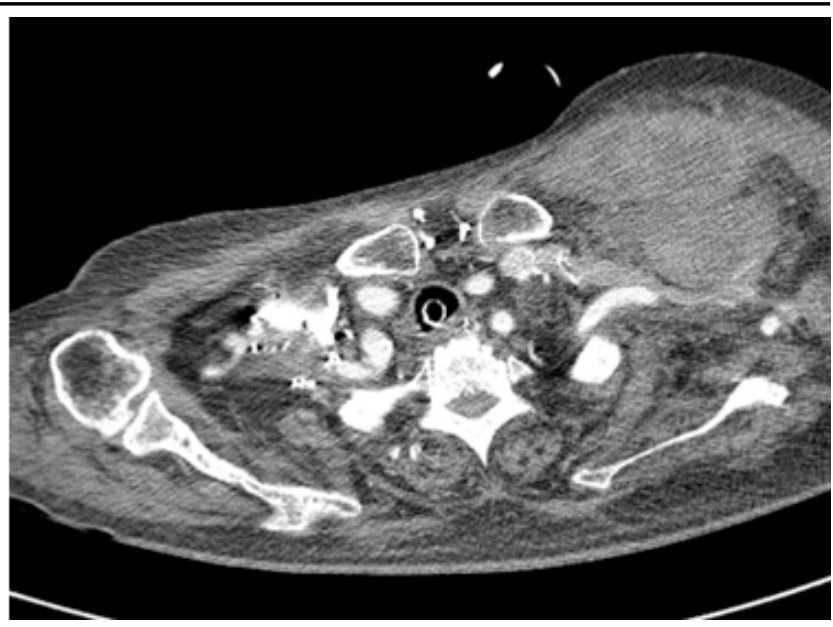

(B)

Figure 1a. CT scanningrevealed a 2.6-cm enhancing Figure $1 \mathrm{~b}$. CT scanning after embolization revealed no nodular lesion, suggesting pseudoaneurysm at the left bleeding and a smaller hematoma. subclavicular area with surrounding hematoma(arrow).

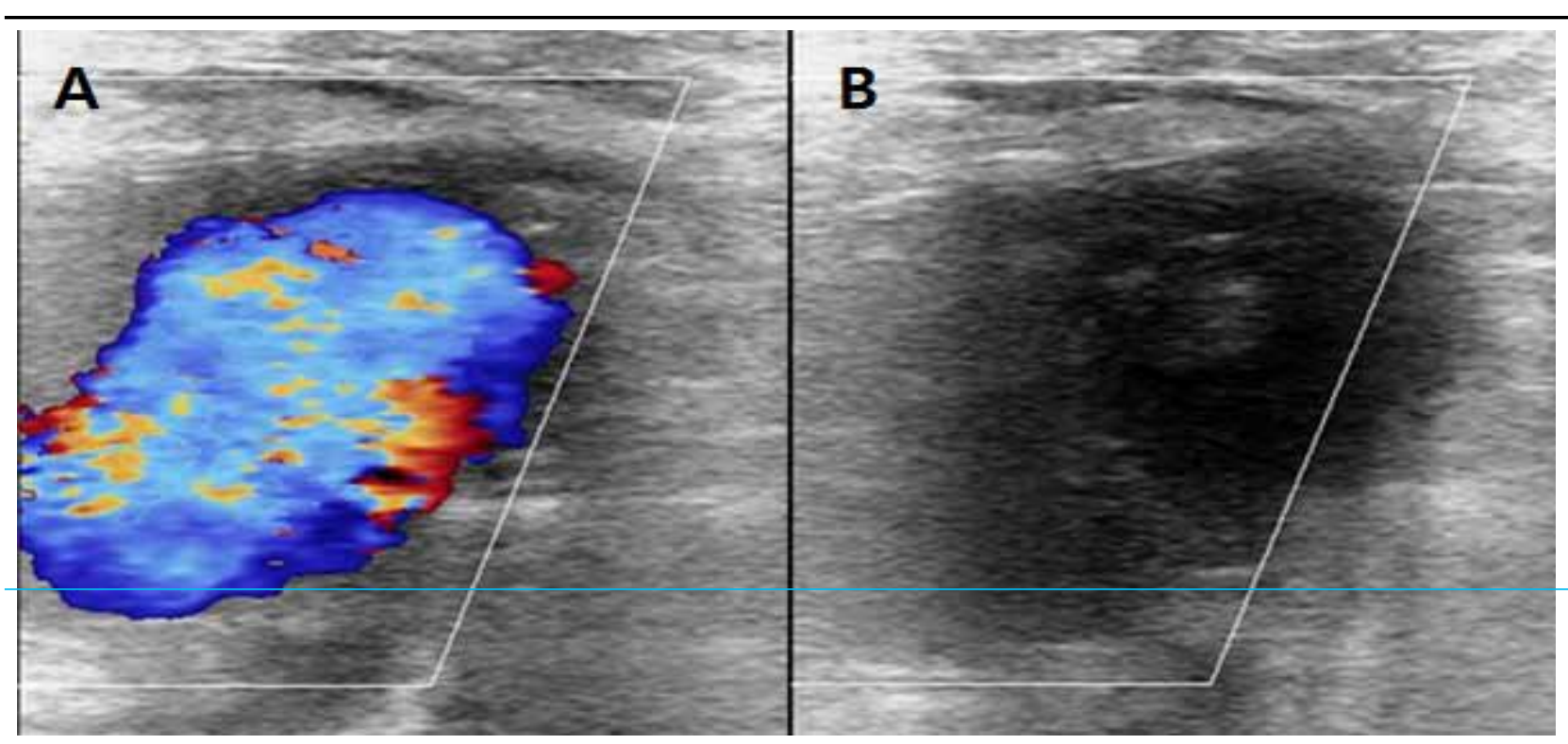

Figure 2. An ultrasound-guided thrombin injection was performed at the pseudoaneurysm of the left subclavian artery (a) pre-injection, (b) post-injection).

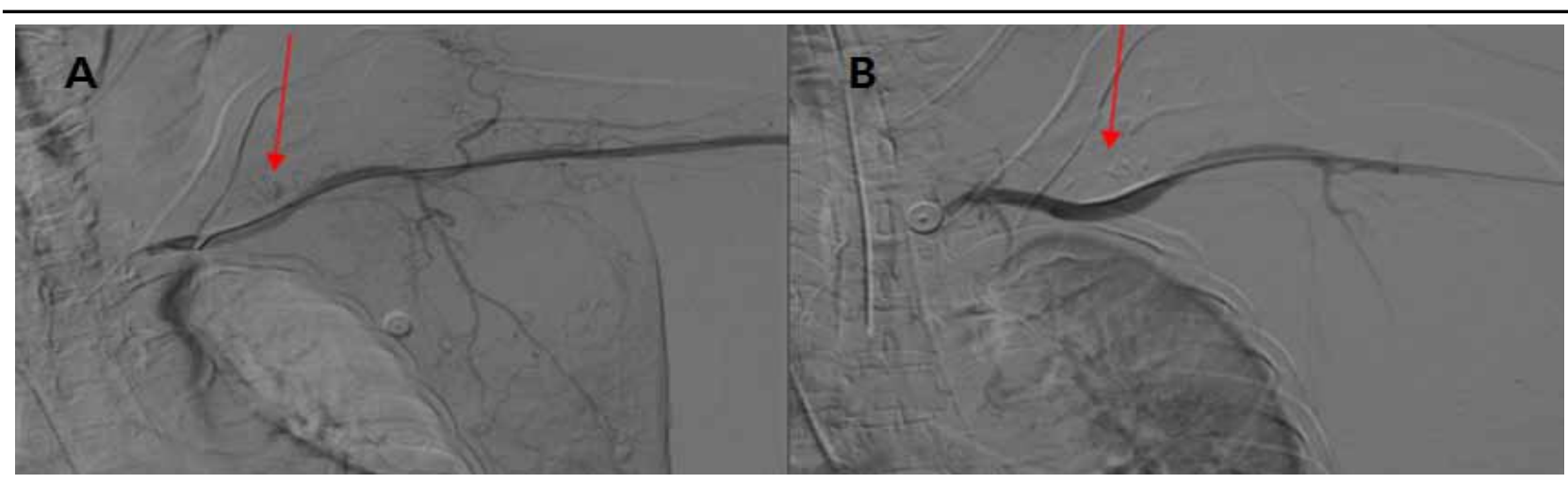

Figure 3. (a) Angiography showed active bleeding of a branch of the leftsubclavian artery(arrow). (b) After embolization, angiography showed non-visualization of extravasation of contrast media(arrow). 


\section{REFERENCES}

1. David C. McGee, M.D., and Michael K. Gould, M.D. Preventing Complications of Central Venous Catheterization. N Engl J Med 2003;348:1123-33.

2. Schwengel DA, McGready J, Berenholtz SM, Kozlowski LJ, Nichols DG, Yaster M. Peripherally inserted central catheters: a randomized, controlled prospective trial in pediatric surgical patients. Anesth Analg. 2004;99:1038-43.

3. Piffaretti G, Tozzi M, Lomazzi C, Rivolta N, Caronno R, Laganà $\mathrm{D}$ et al. Endovascular treatment for traumatic injuries of the peripheral arteries following blunt trauma. Injury. 2007 Sep;38(9):1091-7.

4. Hilary A. Brown, John E. Aruny, John A. Elefteriades, , Bauer E. Sumpio. Subclavian Aneurysm Presenting with Massive Hemoptysis: A Case Report and Review of the Literature Int J Angiol 2013;22:69-74.

5. Jeganathan R1, Harkin DW, Lowry P, Lee B. Iatrogenic subclavian artery pseudoaneurysm causing airway compromise: treatment with percutaneous thrombin injection. J Vasc Surg. 2004 Aug;40(2):371-4.
6. Boccardo P, Remuzzi G, Galbusera M. Platelet dysfunction in renal failure. 2004 Oct;30(5):579-89.

7. Ohki T, Veith FJ. Five-year experience with endovascular grafts for the treatment of aneurysmal, occlusive and traumatic arterial lesions. Cardiovasc Surg. 1998;6(6):552-65.

8. Marin ML, Hollier L, Avrahami R, Parsons R. Varying Strategies For Endovascular Repair Of Abdominal And Iliac Artery Aneurysms. Surg Clin North Am. 1998;78(4):631-45.

9. La Perna L, Olin JW, Goines D, Childs MB, Ouriel K. Ultrasound-guided thrombin injection for the treatment of postcatheterization pseudoaneurysms. Circulation. 2000;102:2391-95.

10. Bukhari HA, Saadia R, Hardy BW. Urgent endovascular stenting of subclavian artery pseudoaneurysm caused by seatbelt injury. Can J Surg.2007 Aug;50(4):303-4. 